\title{
Point/Counterpoint Debate
}

\section{Leptin for type 1 diabetes: coming onto stage to be (or not?)}

Oral EA. Leptin for type 1 diabetes: coming onto stage to be (or not?). Pediatric Diabetes 2012: 13: 68-73.

The discovery of the adipocyte hormone leptin completely changed our view of energy metabolism. In addition, the discovery of leptin rapidly progressed to clinical development. After a decade of clinical studies, leptin appears not to be the magic bullet therapy for obesity; however, it has a robust role in rare human conditions characterized by its deficiency. Recent exciting work from the Unger laboratory suggests that leptin therapy may also have a potential role for the treatment of Type 1 diabetes. In this review we discuss the positive evidence why such an approach is worthwhile. In order to achieve this broad goal, we reviewed available literature and provided our interpretation of the evidence presented in the original research papers. The potential cautionary aspects of this novel approach will be discussed in an accompanying article.

\author{
Elif A Oral \\ Metabolism, Endocrine and Diabetes \\ Division (MEND), University \\ of Michigan, Ann Arbor, MI, USA \\ Key words: autoimmunity - glucagon \\ - insulin - lipotoxicity - therapeutics \\ Corresponding author: \\ Elif A Oral, \\ Metabolism, Endocrine and Diabetes \\ Division (MEND), \\ University of Michigan, \\ MSRB II, 5570C \\ Ann Arbor, MI, \\ USA. \\ Tel: (734) 615-7271; \\ fax: (734) 936-6684; \\ e-mail: eliforal@umich.edu
}

Submitted 20 April 2011. Accepted

for publication 10 May 2011
Exactly 72 years after the discovery of insulin, a hormone that offered life full of potential to all paitents with type 1 diabetes, an equally groundbreaking discovery of the adipocytokine, leptin, completely changed our understanding of energy homeostasis in mammals. This pivotal discovery has granted a new status to adipose tissue as an endocrine organ, promoted the central nervous system to the level of master regulator of human metabolism, and quickly led to the development of leptin analogs as potential human therapies. Although the original focus was exploration of leptin as a potential therapy for human obesity, both animal and clinical data in multiple disease states provide strong support for a leptin analog as a potent physiological replacement therapy in states of 'leptin deficiency'. While trying to understand the therapeutic roles for leptin, two important facts need to be kept in mind: (i) leptin is a pleiotropic hormone with many regulatory functions and (ii) the clinical applicability of leptin is likely wider than absolute deficiency. Anchoring onto these facts, novel therapeutic potentials for leptin are being sought in other disease states.
Among these, type 1 diabetes stands out as an area for which there is a lot of enthusiasm, leading to exciting, newly piloted human trials, which comprise the translational extension of a recently published study by Wang and Unger (1). The purpose of this article is to summarize the basic and clinical data that provide support to exploration of leptin as an adjunctive therapy for patients suffering from type 1 diabetes. I will also present our vision of how leptin therapy may be utilized in the clinical setting of type 1 diabetes. Clearly, far more data are needed to define the precise role of this remarkable hormone as therapy in type 1 diabetes.

\section{A brief review of leptin physiology}

Since the story of leptin therapeutics begins with the discovery of leptin, it is useful to briefly review the physiology of leptin. A protein structurally similar to cytokines, leptin is the protein product of the $o b$ gene in mice and humans (2). The main site of leptin synthesis is adipose tissue (white more than brown, subcutaneous more than visceral) and blood levels of leptin correlate 
with total body fat. Leptin's main function is thought to be as an energy sensor via informing the brain of the energy storage level of the body(3). In response to this signal, the brain makes appropriate adjustments to change food intake and energy expenditure to re-establish the energy homeostasis. The $o b / o b$ mice, which have complete deficiency of leptin, are hyperphagic, hypothermic, and morbidly obese, indicating increased energy intake with reduced energy expenditure (3). Treating ob/ob mice with leptin causes a decrease in appetite and promotes weight loss, the majority of which is the total body fat. These mice also have a marked hepatic steatosis coupled with hepatic insulin resistance and hyperglycemia. In addition, the $o b / o b$ mice are infertile and have a number of other hypothalamic pituitary axes abnormalities such as central hypothyroidism, hypercorticosteroenemia due to central corticotropin releasing factor (CRF) neuron activation, and central linear growth impairment. More recently, immunological abnormalities such as decreased CD4 counts, impaired activation of peripheral blood mononuclear cells and a protective effect against permissive autoimmunity have been observed in the murine $o b / o b$ model. Replacement of leptin in the $o b / o b$ mouse significantly reverses or corrects all these abnormalities. As such, leptin is viewed now not as a simple signal that mediates eating behavior, but rather an integrative control switch, signaling status of energy stores to the brain over a period of days to weeks that in turn controls adaptive response to a state of low energy availability.

\section{Why leptin therapy may have theraputic potential in type 1 diabetes}

We have come up with what we believe are the 'Top 10 ' reasons why leptin therapy may have therapeutic potential in type 1 diabetes in Table 1 . It is important to note that we do not foresee that leptin therapy would replace insulin treatment completely. Rather, we foresee a role for leptin as an adjunctive therapy to help 'fine-tune' metabolic control. It could be administered to reduce the number of insulin injections as well as the total daily dose required. It could help smooth out the variability in postmeal excursions as wellas reducing basal insulin doses required to decrease hepatic glucose output. Now that we have provided a list of reasons why leptin therapy may be of benefit in type 1 diabetes and offered a context for its use, let us examine each point closely.

\section{The need for improved therapy in type 1 diabetes}

Type 1 diabetes affects $0.5 \%$ of the general population. Even though we have come a long way in the delivery of insulin analogs to patients, current treatment strategies
Table 1. Top 10 reasons why leptin may have a therapeutic role in type 1 diabetes

\begin{tabular}{|c|c|}
\hline S.No & Reasons \\
\hline 1 & $\begin{array}{l}\text { There is an ongoing need to improve the treatment } \\
\text { plan of type } 1 \text { diabetics. New drugs are needed. }\end{array}$ \\
\hline 2 & $\begin{array}{l}\text { Type } 1 \text { diabetes is a state of 'relative leptin } \\
\text { deficiency'. Replacement of leptin in states of } \\
\text { leptin deficiency is effective. }\end{array}$ \\
\hline 3 & $\begin{array}{l}\text { Leptin has antisteatotic actions in peripheral } \\
\text { tissues. Steatosis, which can be promoted by } \\
\text { insulin therapy, is known to predict and may } \\
\text { even cause cardiovascular death. }\end{array}$ \\
\hline 4 & $\begin{array}{l}\text { Leptin can suppress elevated glucagon levels. } \\
\text { Targeting just elevated glucagon levels can } \\
\text { abrogate the need for insulin in rodents. }\end{array}$ \\
\hline 5 & $\begin{array}{l}\text { Leptin can lead to the restoration of insulin } \\
\text { secretion in islets by reversing lipotoxicity. }\end{array}$ \\
\hline 6 & $\begin{array}{l}\text { Leptin can reduce food intake in non-obese } \\
\text { humans and can oppose insulin-induced weight } \\
\text { gain in rodent models. }\end{array}$ \\
\hline 7 & $\begin{array}{l}\text { Leptin can improve lipid metabolism in ways that } \\
\text { insulin cannot. }\end{array}$ \\
\hline 8 & $\begin{array}{l}\text { Leptin improves insulin sensitivity in human } \\
\text { lipodystrophy. }\end{array}$ \\
\hline 9 & $\begin{array}{l}\text { Leptin ameliorates metabolic control in patients } \\
\text { with autoimmune lipodystrophy and type } 1 \\
\text { diabetes without adverse clinical effects on the } \\
\text { course of the background autoimmune disorder. }\end{array}$ \\
\hline 10 & $\begin{array}{l}\text { Leptin can ameliorate metabolic control in patients } \\
\text { with presumed insulin receptor mutations } \\
\text { carrying the clinical diagnosis of Rabson- } \\
\text { Mendenhall syndrome. Thus, leptin can crosstalk } \\
\text { with insulin-signaling cascade in humans. }\end{array}$ \\
\hline
\end{tabular}

are cumbersome and the majority of patients fall short of desired metabolic control. Even with metabolic control at its best, patients still experience a high variability of glucose levels especially after meals. Patients are constantly threatened by fear of hypoglycemia and weight gain even with near-goal hemoglobin A1c (HbA1c) levels. These latter two conditions continue to contribute to reduced life expectancy in type 1 diabetes as well as reduced quality of life. Current rapid acting insulin analogs peak their action within $2 \mathrm{~h}$ and work for about $4 \mathrm{~h}$. The normal pancreas on the other hand is capable of responding to a meal challenge by rapid insulin release and getting it cleared by $50 \%$ within $5 \mathrm{~min}$. The delayed onset of subcutaneously injected insulin results in immediate postmeal glucose excursions followed by a rapid drop in blood glucose as insulin becomes available. Any delayed effect of food on glycemia caused by the ingestion of lipids or amino acids are typically not accounted for by meal bolus dosing.

Another imperfection in current insulin-dosing regimens results from the discordance between sensitivity thresholds in insulin signaling to regulate lipogenesis vs. gluconeogenesis in the liver. The basal doses of insulin typically used in clinical practice are still able to promote lipogenesis, while raising the threshold for 


\section{Oral}

regulation for hepatic glucose output. Understanding this paradox would help understand why weight gain can occur while diabetes is still poorly controlled and why weight gain almost always accompanies improved glucose control. In search of bettering glycemic control, novel hormonal therapies for type 1 diabetes have emerged such as amylin analogs. One presumed action of the amylin hormone is targeting glucagon oversecretion that is known to be a key metabolic feature of insulin deficiency. Therefore, other additional therapeutic targets such as leptin (see evidence below) that affect glucagon oversecretion or which alter postmeal lipid or amino-acid handling may have a place in more modern type 1 diabetes regimens. Furthermore, leptin can simply reduce food intake and thus create an effect by reducing the 'meal-burden' and providing a net positive effect on glycemic control.

Type 1 diabetes: a state of 'relative insulin deficiency'

The real success of leptin therapy has been observed in the adaptation of hormone replacement strategy in states of leptin deficiency because low leptin levels appear to provide an integrative signal of energy deficit for the organism. Reduced food availability and empty adipocyte stores lead to the low leptin signal. Insulin deficiency in type 1 diabetes causes a state of increased lipolysis from the adipocytes which increases circulating free fatty acids and eventually ketonemia. Both these metabolites can reduce adipocyte's ability to secrete leptin, signaling an 'energy deficit'. Therefore, type 1 diabetes is one of the conditions that we have begun to call 'relative leptin deficiency' to begin to describe conditions which may be amenable to leptin repletion strategy. Aside from type 1 diabetes, sleep deprivation, Circadian shifts, hypothalamic amenorrhea, genetic mechanisms that interfere with leptin secretion, and variations in body fat depots such as partial lipodystrophy syndromes or loss of subcutaneous fat compartments are among other conditions characterized by 'relative leptin deficiency'. In our opinion, the repletion strategy to be used in the treatment of relative leptin deficiency is analogous to the recently published experience from heterozygote $o b$ mice (4).

\section{Leptin has strong antisteatotic effects}

Steatosis is the deposition of lipids inside tissues outside the adipose tissue. It usually occurs due to the availability of excess lipids for storage above and beyond what the adipose compartment can store. Thus, steatosis is a complication of obesity, but is also seen as a result of lipodystrophy (a human condition characterized by a paucity of fat either through acquired or genetic reasons). Insulin can promote steatosis in the liver and is a long-term complication of insulin therapy. From a therapeutic perspective, it is important to try to target steatosis because the presence of fat in the muscle and liver is associated with an increased risk of cardiovascular disease. Hepatic steatosis is now regarded as at least a forecaster of future cardiovascular events and may even play a causative role.

Leptin replacement strategy leads to resolution of steatosis in the liver and muscle of both $o b / o b$ mice as well as multiple models of leptin-deficient lipodystrophy. Similarly, humans with lipodystrophy respond to leptin therapy with a remarkable resolution of steatosis both in the liver and in muscle. In a mouse model of lipodystrophy, resolution of steatosis in response to physiological leptin replacement was evident after 3 weeks of therapy (5). Furthermore, this study showed that lipogenesis was dysregulated in the liver in the absence of leptin, which was corrected with leptin replacement. To elucidate the mechanism by which leptin reduces hepatic lipid content in livers, Cohen et al. used microarrays to identify genes in the liver that were differentially regulated by leptin or by food restriction (pair-feeding). The gene encoding stearoyl-CoA desaturase (SCD-1) ranked the highest in this analysis (6). The microsomal enzyme SCD-1 is required for the biosynthesis of the monounsaturated fats palmitoleate and oleate from saturated fatty acids. Additionally, leptin increases hepatic and adipocyte expression of PPAR- $\alpha$ coactivator- $1 \alpha$ (7) which regulates mitochondria biogenesis and fat oxidation.

Another important antisteatotic effect of leptin was uncovered by studying muscle tissue. Minokoshi and colleagues showed that leptin selectively stimulates phosphorylation and activation of the $\alpha 2$ catalytic subunit of AMPK in skeletal muscle, thus establishing a previously unknown signaling pathway for leptin (8). In parallel with its activation of AMPK, leptin suppresses the activity of acetyl-CoA carboxylase, thereby stimulating the oxidation of fatty acids in muscle.

These antistetaotic effects of leptin which are peripheral endpoints are likely mediated via its central action and not directly through its peripheral receptors. The lipopenic action of leptin has been duplicated in rodents with small, centrally administered doses (9). The effect is achieved even with the knockout of peripheral leptin receptors (10) and this suggests that leptin signals through one or more centrally mediated intermediates, which in turn lead to increased fatty acid oxidation and suppression of lipogenesis.

\section{Leptin can suppress glucagon levels and hyperglucagonemia is a hallmark feature of type 1 diabetes}

Studies emerging from the laboratory of Roger Unger over the last 35 years have suggested that 
hyperglucagonemia is a key characteristic of the diabetic state and the hepatic actions of glucagon after meals to stimulate hepatic glucose output accounted for most of the postmeal excursions in glucose levels. As shown in the paper by Wang and Unger in 2010, leptin therapy reversed the diabetic state of multiple models of insulin deficiency and abrogated the need for insulin therapy (1). The authors have suggested that these actions of leptin were related to a reduction in plasma glucagon levels. More recently, the same laboratory has published that glucagon receptor-deficient mice treated with streptozotocin (STZ) did not develop a need for insulin therapy despite losing insulin secretion (11). These results were supported in a study by Hancock et al. that mice, lacking alpha cells, were also protected from clinical diabetes despite STZ treatment and loss of insulin secretion (12). Such observations led the Unger lab to posit that insulin would have no role in a liver not exposed to the action of glucagon and leptin appeared to be one of the key regulators of glucagon secretion.

\section{Leptin reduces food intake in non-obese humans} and opposes insulin-induced weight gain observed in models of type 1 diabetes

Leptin therapy leads to a reduction in food intake in non-obese humans such as women with hypothalamic amenorrhea (13) and in patients with lipodystrophy (14) as well as a few obese humans with mutations in the leptin gene (15). This is usually in parallel to a reduction in body weight, with predominant fat loss as opposed to lean mass. A similar effect was observed in obese humans who were treated with leptin alone or in combination with Pramlintide (16). Thus, leptin would be expected to lower food intake and decrease the overall meal load. Historically, prior to the discovery of insulin, the treatment for type 1 diabetes was caloric restriction and forced starvation. Leptin therapy may in some ways simulate such a state by signaling a decreased need for food (i.e., Being 'content'), reducing the need for insulin.

In the leptin-treated mouse models of type 1 diabetes, limitation of food intake by pair-feeding animals without leptin administration did not provide the extent of benefit noted on glucose and lipid parameters, and liver weight, suggesting that leptin's effect on metabolism is not totally dependent on limiting food intake (1). As expected, leptin therapy led to a decrease in body weight primarily from the body fat compartment but also lowered hepatic fat depots, while insulin therapy led to an increase in body weight as well as an increase in hepatic fat content.

\section{Leptin has unique effects on lipid metabolism}

In the above sections, we have touched upon distinctive effects of leptin on lipid metabolism. Leptin reverses steatosis uniquely. It promotes beta-oxidation of fatty acids in both muscle and liver. It can also have consequences in the adipose tissue either directly or through lowering glucagon, to switch the metabolic pathways toward oxidation and inhibiting lipogenesis. Leptin has a regulatory action on key transcriptional factors such as steroid regulatory element binding protein 1 (SREBP1) and SCD1 as well as PPAR- $\alpha$. Unger and Wang have also demonstrated an improved cholesterol profile of diabetic mice upon treatment with leptin (1). Leptin also lowers triglyceride levels in this setting as well as in rodents and humans with lipodystrophy. The vast amount of available data in the literature underscores that leptin and insulin have parallel central nervous system effects to regulate glucose metabolism and food intake, while their effects on lipid metabolism are distinctive.

Leptin can lead to restoration of insulin secretion by opposing 'lipotoxic' effects on islet cells

From 1995 to 2002, the Unger laboratory focused their efforts in defining the phenomenon of lipotoxicity (17). This is a term to describe the consequences of lipid spillover to tissues outside the adipocytes such as liver, muscle, myocardium, and islets. Accumulation of excessive lipid in the islets were reported to cause ceramide accumulation, increased fibrosis, and apoptosis. Restoration of leptin signaling or leptin repletion successfully recovered islet cell insulin secretion and reversed diabetes in multiple rodent models of obesity, leptin resistance, or leptin deficiency (17). These findings add to the characteristics of leptin that make it an attractive tool for the treatment of type 1 diabetes.

\section{Leptin works as an insulin-sensitizer in vivo} in some human conditions characterized by leptin-deficiency such as severe lipodystrophy

In 2000, about 6 years after the discovery of leptin, we began the first leptin replacement trial in patients with severe lipodystrophy (14). The goal of these studies was physiologic replacement, analogous to using insulin in type 1 diabetes, because in both disease states the organs that manufactures the replaced hormone are functionally absent. In a short period such as a few months, patients with the most severe forms of fat deficiency and most pronounced metabolic defects showed a remarkable improvement in their insulin sensitivity and dyslipidemia. An average of $1.5-2 \%$ reduction in HbAlc has remained constant at 4and 12-month time points after initiation of leptin throughout a decade of ongoing studies (18). The improvement in insulin sensitivity was apparent in liver as well as in muscle compartments. These studies 
demonstrated for the first time that leptin had insulinsensitizing actions in humans and that leptin accounted for most of the metabolic effects of adipose tissue.

\section{Leptin enhances metabolic control in humans with autoimmune lipodystrophy and type 1 diabetes}

Among lipodystrophic patients who were treated with leptin, there were at least three patients who had clear evidence for autoimmune lipodystophy and type 1 diabetes ((19) and unpublished observations). In these selected patients, it is reassuring that leptin led to significant metabolic improvements such as reductions in $\mathrm{HbAlc}$, triglycerides, food intake (also with reduction in anxiety associated with constantly seeking food), and improvement in hepatic steatosis. None of the patients experienced deterioration in their underlying autoimmune diseases. It is also important to point out that none of these patients were able to discontinue insulin therapy completely, but their insulin dosages were reduced from heroic doses down to weight-consistent ranges. They were able to reduce the number of insulin injections required to maintain normoglycemia. All three patients expressed satisfaction with their regimen and attributed the satisfaction to reduction in hepatomegaly (which was impeding their mobility) as well as improved satiety.

\section{Leptin helps to improve metabolic control in patients with presumed insulin receptor mutations}

We also had the opportunity to treat and observe the metabolic effects caused by leptin therapy in two siblings who had the clinical features of RabsonMendenhall syndrome with severe hyperinsulinemia, acanthosis nigricans, hyperglycemia, short stature, and multiple dysmorphic features (20). These patients (even though not studied) have presumed mutations of their insulin receptor gene. They also displayed relatively low levels of circulating leptin $(3-4 \mathrm{ng} / \mathrm{dL})$ and thus were treated with recombinant leptin now for nearly 10 years. In both these patients, leptin therapy helped to decrease insulin doses and improve glucose control, decreasing glucosuria and allowing improved growth. Leptin therapy was withdrawn in a controlled setting on two separate occasions over the observation period and both these periods were associated with worsening metabolic control and a dramatic increase in insulin dosages. The effects observed in these two patients comprise the most direct evidence that insulin and leptin signaling crosstalk to regulate glucose metabolism in humans.

\section{Conclusions}

In conclusion, the available preclinical evidence studied mostly in the Unger laboratory over a 15 -year-time period coupled with results available from the translational studies obtained in humans with various forms of leptin deficiency and diabetes, support the notion that it is worthwhile to undertake clinical trials to test the effects of leptin in patients with type 1 diabetes. As mentioned previously and as suggested by our limited experience in patients with type 1 diabetes and acquired lipodystrophy, we do not foresee that leptin can replace insulin therapy in type 1 diabetes. It can, however, have a strong adjunctive role. As human studies are being undertaken, certain areas of caution (which are mentioned in an accompanying paper) will have to be carefully weighed and incorporated into the study design with appropriate safety measures.

\section{References}

1. Wang MY, Chen L, Clark GO et al. Leptin therapy in insulin-deficient type I diabetes. Proc Natl Acad Sci U S A 2010: 107: 4813-4819.

2. Zhang Y, Proenca R, Maffei M, Barone M, Leopold L, Friedman JM. Positional cloning of the mouse obese gene and its human homologue. Nature 1994: 372: 425-432.

3. Friedman JM, HalaAs JL. Leptin and the regulation of body weight in mammals. Nature 1998: 395: 763-770.

4. Begriche K, Letteron P, Abbey-Toby A et al. Partial leptin deficiency favors diet-induced obesity and related metabolic disorders in mice. Am J Physiol Endocrinol Metab 2008: 294: E939-E951.

5. Shimomura I, Hammer RE, Ikemoto S, Brown MS, GoldSTEIN JL. Leptin reverses insulin resistance and diabetes mellitus in mice with congenital lipodystrophy. Nature 1999: 401: 73-76.

6. Cohen P, Miyazaki M, Socci ND et al. Role for stearoyl-CoA desaturase-1 in leptin-mediated weight loss. Science 2002: 297: 240-243.

7. Puigserver P, Spiegelman BM. Peroxisome proliferator-activated receptor-gamma coactivator 1 alpha (PGC-1 alpha): transcriptional coactivator and metabolic regulator. Endocr Rev 2003: 24: 78-90.

8. Minokoshi Y, Kim YB, Peroni OD et al. Leptin stimulates fatty-acid oxidation by activating AMPactivated protein kinase. Nature 2002: 415: 339-343.

9. Asilmaz E, Cohen P, Miyazaki M et al. Site and mechanism of leptin action in a rodent form of congenital lipodystrophy. J Clin Invest 2004: 113: 414-424.

10. Cohen P, Zhao C, Cai X et al. Selective deletion of leptin receptor in neurons leads to obesity. J Clin Invest 2001: 108: 1113-1121.

11. LeE Y, Wang MY, Du XQ, Charron MJ, Unger RH. Glucagon receptor knockout prevents insulin-deficient type 1 diabetes in mice. Diabetes 2011: 60: 391-397.

12. Hancock AS, Du A, Liu J, Miller M, May CL. Glucagon deficiency reduces hepatic glucose production and improves glucose tolerance in adult mice. Mol Endocrinol 2010: 24: 1605-1614.

13. Welt CK, Chan JL, Bullen J et al. Recombinant human leptin in women with hypothalamic amenorrhea. N Engl J Med 2004: 351: 987-997. 
14. Oral EA, Simha V, Ruiz E et al. Leptin-replacement therapy for lipodystrophy. N Engl J Med 2002: 346: 570-578.

15. Faroogi IS, Matarese G, Lord GM et al. Beneficial effects of leptin on obesity, $\mathrm{T}$ cell hyporesponsiveness, and neuroendocrine/metabolic dysfunction of human congenital leptin deficiency. J Clin Invest 2002: 110: 1093-1103.

16. Ravussin E, Smith SR, Mitchell JA et al. Enhanced weight loss with pramlintide/metreleptin: an integrated neurohormonal approach to obesity pharmacotherapy. Obesity (Silver Spring) 2009: 17: 1736-1743.

17. Unger RH. The physiology of cellular liporegulation. Annu Rev Physiol 2003: 65: 333-347.
18. Oral EA, Chan JL. Rationale for leptin-replacement therapy for severe lipodystrophy. Endocr Pract 2010: 16: 324-333.

19. Park JY, Chong AY, Cochran EK et al. Type 1 diabetes associated with acquired generalized lipodystrophy and insulin resistance: the effect of long-term leptin therapy. J Clin Endocrinol Metab 2008: 93: 26-31.

20. Cochran E, Young JR, Sebring N, DePaoli A, Oral EA, Gorden P. Efficacy of recombinant methionyl human leptin therapy for the extreme insulin resistance of the Rabson-Mendenhall syndrome. J Clin Endocrinol Metab 2004: 89: 1548-1554. 